\title{
Dual Strategic Approach to Prepare Defluorinated Triazole Embedded Covalent Triazine Frameworks with High Gas Uptake Performance
}

Soumya Mukherjee, ${ }^{a b}$ Monojit Das, ${ }^{a b}$ Anupam Manna, ${ }^{a b}$ Rajamani Krishna, ${ }^{c}$ and Sanjib $\operatorname{Das}^{*} a b$

${ }^{a}$ Department of Colloids \& Materials Chemistry, CSIR-Institute of Minerals \& Materials Technology, Bhubaneswar 751013, India.

${ }^{b}$ Academy of Scientific \& Innovative Research (AcSIR), Ghaziabad 201002, India.

${ }^{c}$ Van't Hoff Institute for Molecular Sciences, University of Amsterdam, Science Park 904, 1098 XH Amsterdam, The Netherlands.

*Corresponding Author: sanjibdas@immt.res.in

\begin{tabular}{|c|c|c|}
\hline Section & Contents & Pages \\
\hline $\mathbf{A}$ & Materials and Methods & $02-03$ \\
\hline B & General Synthetic Procedure of Precursors & $03-04$ \\
\hline $\mathbf{C}$ & ${ }^{1} \mathrm{H}$ and ${ }^{19} \mathrm{~F}$ NMR Spectra & $04-06$ \\
\hline D & Elemental Analysis & 06 \\
\hline $\mathbf{E}$ & XPS Survey Scan & 06 \\
\hline $\mathbf{F}$ & Wide-Angle Powder XRD Analysis & 07 \\
\hline $\mathbf{G}$ & FE-SEM and HRTEM Analysis & 07 \\
\hline $\mathbf{H}$ & Pore Size Distribution Curves Derived from $\mathrm{CO}_{2}$ Isotherm & 07 \\
\hline $\mathbf{I}$ & Fitting of Unary Isotherm Data & 08 \\
\hline $\mathbf{J}$ & Isosteric Heat of Adsorption & 08 \\
\hline $\mathbf{K}$ & IAST Calculation of Component Uptakes and Adsorption Selectivities & 09 \\
\hline $\mathbf{L}$ & Transient Breakthrough of mixtures in Fixed Bed Adsorber & 10 \\
\hline M & Henry Selectivity Studies & 10 \\
\hline $\mathbf{N}$ & Henry Plot for $\mathrm{CO}_{2}, \mathrm{~N}_{2}$, and $\mathrm{CH}_{4}$ & 11 \\
\hline $\mathbf{O}$ & Productivity for $\mathrm{CO}_{2} / \mathrm{CH}_{4}$ Separation: & 11 \\
\hline $\mathbf{P}$ & Comparison Tables of Textural and Gas Uptake Properties & $11-15$ \\
\hline $\mathbf{Q}$ & High Pressure $\mathrm{H}_{2}$ Adsorption Isotherms & $15-16$ \\
\hline $\mathbf{R}$ & Supporting References & $16-19$ \\
\hline
\end{tabular}




\section{Section A. Materials and Methods:}

All chemical reagents were purchased from commercial sources and used as received unless otherwise stated. Periodic acid, potassium iodide, triethylamine, cesium fluoride, copper sulphate pentahydrate and sodium thiosulfate were purchased from Spectrochem, India. 1,3,5-trifluorobenzene, 1,3,5-tribromobenzene, pentafluorobenzonitrile, (trimethylsilyl)acetylene, tetrakis(triphenylphosphine)palladium(0) and sodium ascorbate were purchased from Alfa-Aesar. Anhydrous zinc chloride was purchased from Alfa-Aesar and further dried at $110{ }^{\circ} \mathrm{C}$ under vacuum prior to use. Column chromatography was performed using silica gel purchased from Spectrochem, India. All organic solvents were procured from Spectrochem, India. Anhydrous THF was prepared by freshly distilled from continuously refluxing THF over sodium under argon. Triethylamine was purified prior to use via distillation over $\mathrm{KOH}$.

Solution ${ }^{1} \mathrm{H}$, and ${ }^{19} \mathrm{~F}$ NMR spectra were recorded on a Bruker ${ }^{\circledR}$ Ultrashield instrument operating at a frequency of $400 \mathrm{MHz}$ with tetramethylsilane (TMS) as an internal standard using deuterated $\mathrm{CDCl}_{3}$ or DMSO as solvent. All measurements were carried out at ambient temperature and chemical shifts are reported in parts per million (ppm) relative to the deuterated solvent. Fourier-transform Infrared spectra (FT-IR) of starting materials and assynthesized Tz- $d f$-CTFs materials were obtained using a Perkin Elmer Spectrum-GX spectrophotometer with $\mathrm{KBr}$ pallet in transmittance mode. All FT-IR spectra were background corrected. Powder X-ray diffraction (PXRD) patterns were recorded on a Rigaku Ultima-IV diffractometer using monochromatic $\mathrm{Cu}-\mathrm{K}_{\alpha}(\lambda=1.54060 \AA)$ radiation with a scan speed of $2^{\circ} \mathrm{min}^{-1}$ and a step size of $0.01^{\circ}$ in $2 \theta$. The X-ray tube operated at a voltage of 40 $\mathrm{kV}$ and a current of $30 \mathrm{~mA}$. Thermogravimetric analyses were carried out in a nitrogen stream using Netzsch STA 449F3-jupiter thermogravimetric-differential scanning calorimetry (TG-DSC) analyzer with a heating rate of $10^{\circ} \mathrm{C} / \mathrm{min}$. The Raman spectra were measured on a Horiba LabRAM HR confocal micro-Raman system with an excitation source of $532 \mathrm{~nm}$ diode-pumped solid-state laser. Field emission scanning electron microscopy (FE-SEM) images were taken using Carl Zeiss AG Supra Gemini 55 at an accelerating voltage of $15 \mathrm{kV}$ and equipped with OXFORD energy dispersive X-ray spectrometer. The powder samples were dispersed in ethanol and the suspension was drop cast on a clean piece of silicon wafer. High resolution transmission electron microscopy (HR-TEM) images were captured using field-emission JEOL, JEM-2100F instrument. The powder samples were dispersed in chloroform and one drop of the suspension was drop cast on a carbon-coated copper grid. 
Elemental analysis (C, H, and N) were performed on a Thermo Scientific Flash 2000 Organic Elemental Analyzer. High resolution X-ray photoelectron spectroscopy (XPS) spectra were recorded with PHI 5000 Versa Probe II XPS with AES module comprising argon ion as well as $\mathrm{C} 60$ sputter guns.

Gas adsorption-desorption experiments (up to 1 bar) were carried out on Quantachrome Autosorb-iQ surface area \& pore size analyzer. Before gas adsorption experiment sample was degassed first at $140^{\circ} \mathrm{C}$ under a dynamic vacuum for 12 hours. Highpurity-grade $\mathrm{He}, \mathrm{N}_{2}, \mathrm{H}_{2}, \mathrm{CO}_{2}$ and $\mathrm{CH}_{4}$ gases were used in all adsorption measurements. The $\mathrm{N}_{2}(77 \mathrm{~K})$ and $\mathrm{H}_{2}(77 \mathrm{~K})$ isotherms were measured using a liquid nitrogen bath. The $\mathrm{CO}_{2}$ and $\mathrm{CH}_{4}$ isotherms measured at $273 \mathrm{~K}$ and $298 \mathrm{~K}$ using a jacketed recirculating dewar containing mixed water and ethylene glycol (1:1 ratio) and the dewar was connected to a chiller (JULABO, FL300, working temperature range: -20 to $+40^{\circ} \mathrm{C}$, temperature stability: $\pm 0.5^{\circ} \mathrm{C}$ ) having circulating methanol to precisely achieve the isothermal condition during measurement. The Brunauer-Emmett-Teller (BET) surface area was calculated over the relative pressure range $0.05-0.3 \mathrm{P} / \mathrm{P}_{0}$ whereas Langmuir surface area calculated taking relative pressure range $0-0.15 \mathrm{P} / \mathrm{P}_{0}$. Total pore volumes were measured at relative pressure near $0.99 \mathrm{P} / \mathrm{P}_{0}$ while microporosity was estimated by measuring the pore volume at a relative pressure of $0.1 \mathrm{P} / \mathrm{P}_{0}$. Pore size distributions and pore volumes were derived from both $\mathrm{N}_{2}(77$ $\mathrm{K})$ and $\mathrm{CO}_{2}(273 \mathrm{~K})$ adsorption-desorption isotherms using the classical t-plot method as well as non-local density functional theory (NL-DFT) slit-pore model for porous carbon using ASiQwin software package. High pressure gas adsorption analysis was performed using fully automated volumetric gas sorption analyzer namely iSorb-HP1 (Model-iSorbHP1XKRHSPNF100) from Quantachrome Instrument, USA using Polyscience circulator (serial no.1C1482229) to maintain the required isothermal condition during gas adsorption analysis.

\section{Section B. General Synthetic Procedure of Precursors:}

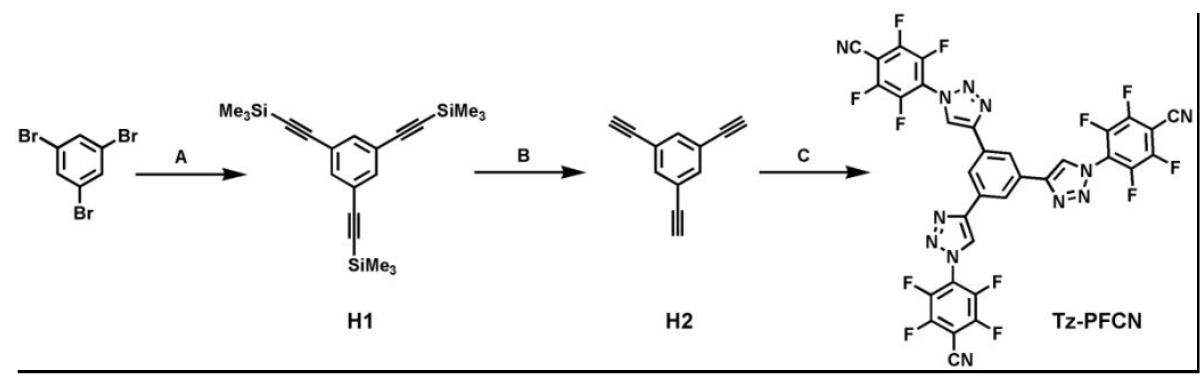

A: Trimethylsilylacetylene, CuI, $\mathrm{Pd}\left(\mathrm{PPh}_{3}\right)_{4}$, THF, Triethylamine, $70^{\circ} \mathrm{C}$, Overnight; B: CsF, Ethanol, Tetrahydrofuran, $50 \mathrm{~min}, \mathrm{RT}$; C: 4-Azidotetrafluorobenzonitrile, Sodium ascorbate, Water, Tetrahydrofuran, $60^{\circ} \mathrm{C}, 3$ days.

Scheme S1: Synthetic route of Tz-PFCN building block. 
1,3,5-tris[2-(trimethylsilyl)ethynyl]benzene: ${ }^{\mathrm{s} 1}$ A $250 \mathrm{ml}$ two neck round bottom flask equipped with stir bar was charged with 1,3,5-tribromobenzne (6.0 g, $19.05 \mathrm{mmol}), \mathrm{CuI}$ (145.12 mg, $0.762 \mathrm{mmol}$ ), $\mathrm{PPh}_{3}$ (999.32 mg, $\left.3.81 \mathrm{mmol}\right), \mathrm{PdCl}_{2}\left(\mathrm{PPh}_{3}\right)_{2}$ (668.56 mg, 0.95 $\mathrm{mmol})$, anhydrous THF $(60 \mathrm{ml})$, and triethylamine $(60 \mathrm{ml})$. The mixture was purged with argon over $10 \mathrm{mins}$ at room temperature followed by dropwise addition of trimethylsilylacetylene $(6.74 \mathrm{~g}, 19.05 \mathrm{mmol})$ under inert condition. The reaction mixture was heated to $70^{\circ} \mathrm{C}$ for overnight and cooled down to room temperature. The mixture was filtered and the filtrate was extracted by chloroform $(3 \times 50 \mathrm{ml})$ and washed with water and then dried over anhydrous $\mathrm{MgSO}_{4}$. Organic layer was evaporated to dryness and the crude product was purified via column chromatography (silica gel, hexane) to obtain cream colored solid compound (5.3 g, 80\%). ${ }^{1} \mathrm{H}$ NMR (400 MHz; $\mathrm{CDCl}_{3}$ ): 0.23 (s, 27H), 7.49 (s, 3H).

1,3,5-triethynylbenzene: ${ }^{52}$ A $50 \mathrm{ml}$ round bottom flask equipped with stir bar was charged with 1,3,5-tris[2-(trimethylsilyl)ethynyl]benzene H1 (2 g, $5.45 \mathrm{mmol})$, tetrahydrofuran (30 $\mathrm{ml})$ and ethanol $(15 \mathrm{ml})$ followed by addition of $\mathrm{CsF}(2.60 \mathrm{~g}, 17.18 \mathrm{mmol})$. The reaction mixture was then stirred for 50 minutes under inert condition at RT. It was filtered then and solvent was evaporated under reduced pressure and the crude product was purified by silica gel column chromatography (petroleum ether) to obtain the white colored pure compound $\mathbf{H 2}$ (750 mg, 91.6\% yield). ${ }^{1} \mathrm{H}$ NMR (400 MHz; $\mathrm{CDCl}_{3}$ ): 3.11 (s, 3H), 7.57 (s, 3H).

\section{Section C. ${ }^{1} \mathrm{H}$ and ${ }^{19}$ F NMR Spectroscopy Studies:}

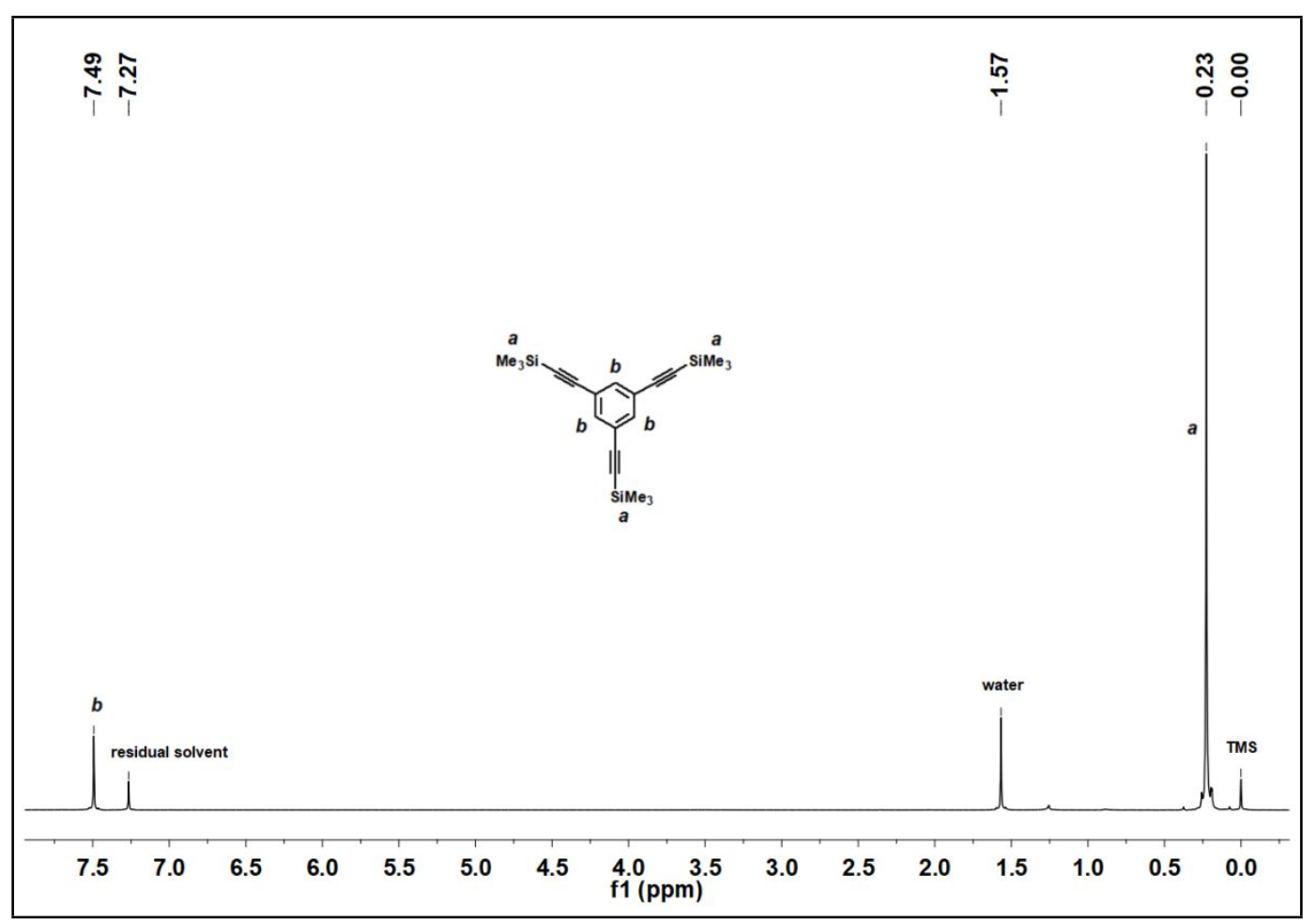

Figure S1: ${ }^{1} \mathrm{H}$ NMR of 1,3,5-tris[2-(trimethylsilyl)ethynyl]benzene 


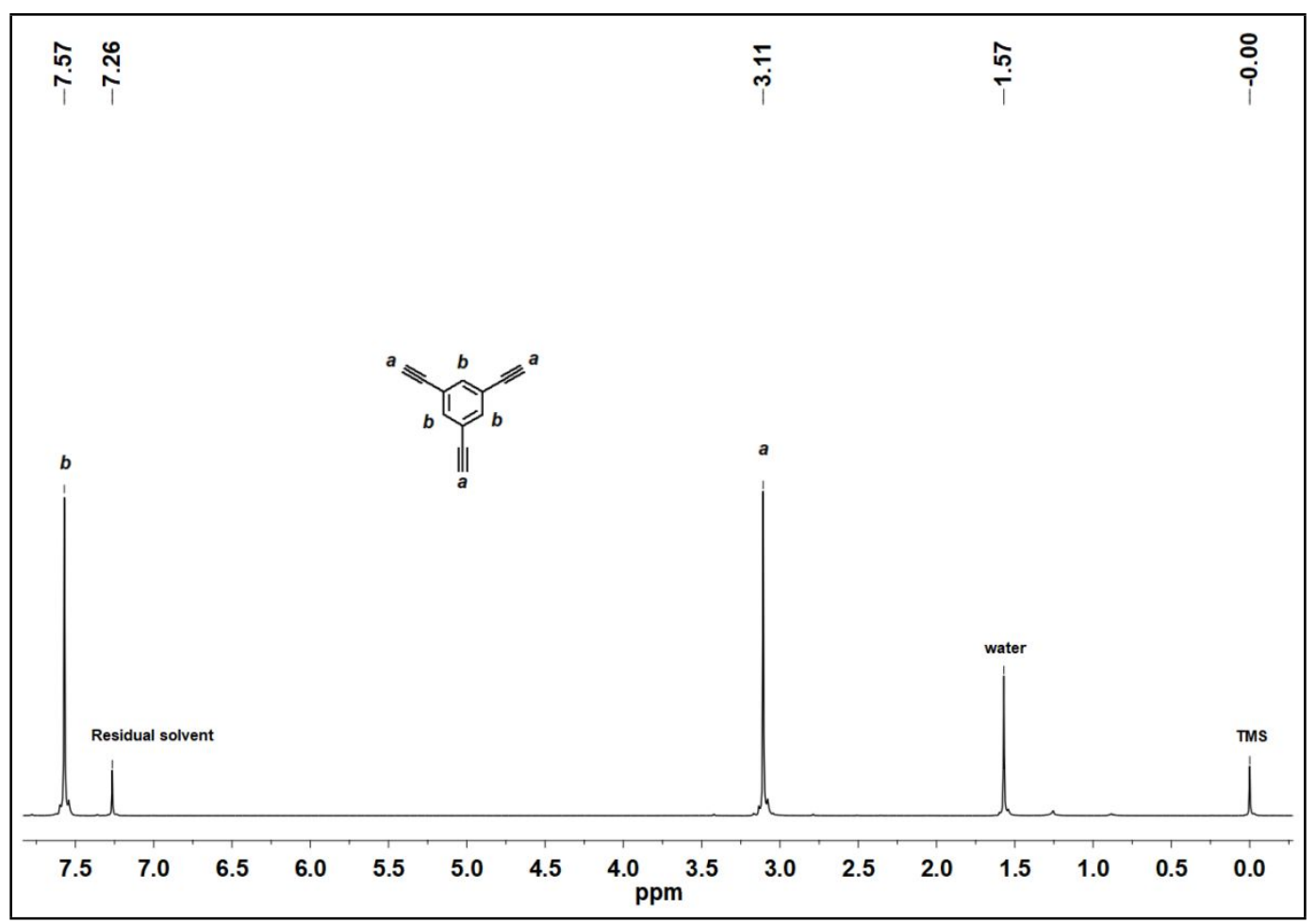

Figure S2: ${ }^{1} \mathrm{H}$ NMR of 1,3,5-triethynylbenzene

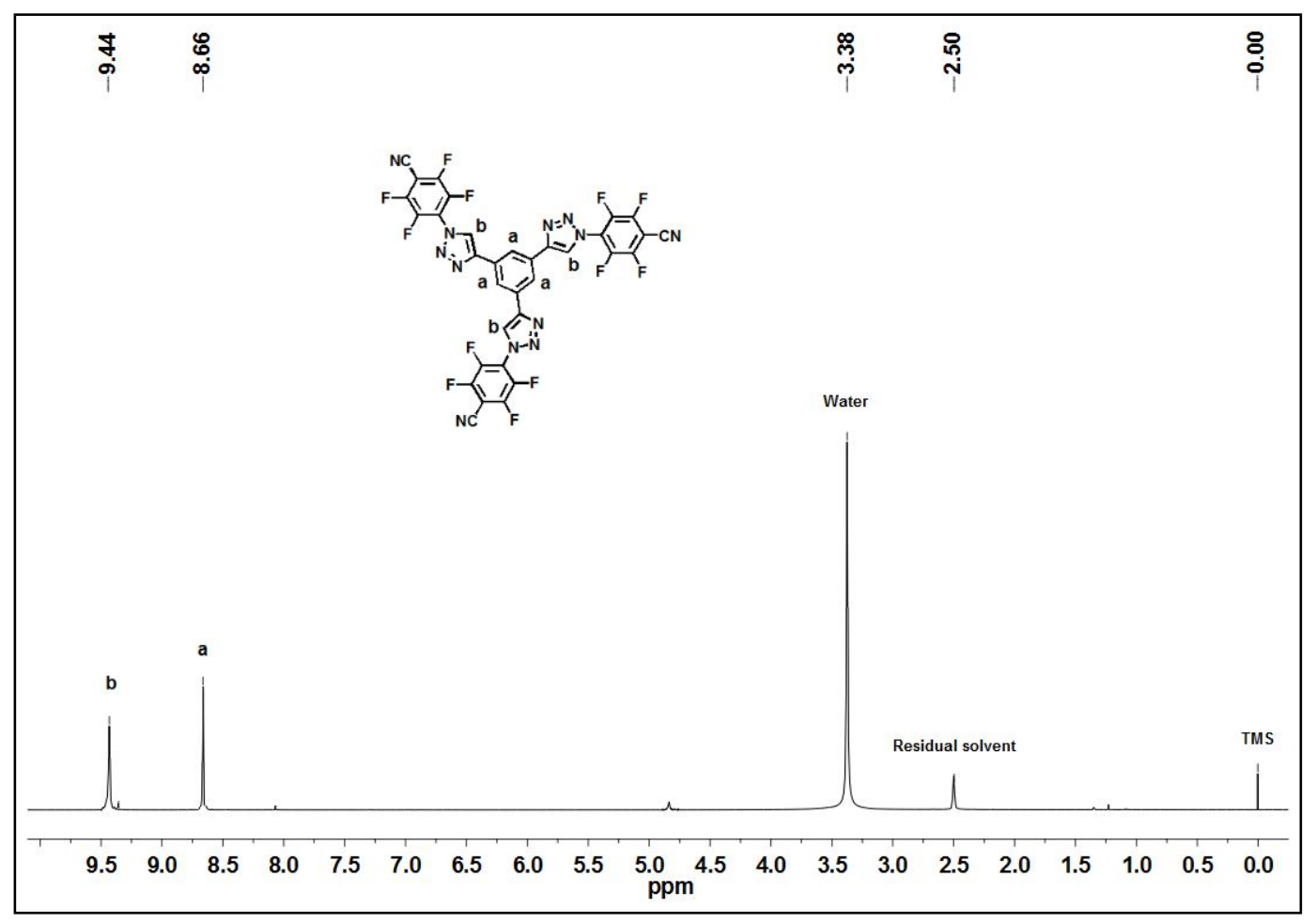

Figure S3: ${ }^{1} \mathrm{H}$ NMR of Tz-PFCN building block 


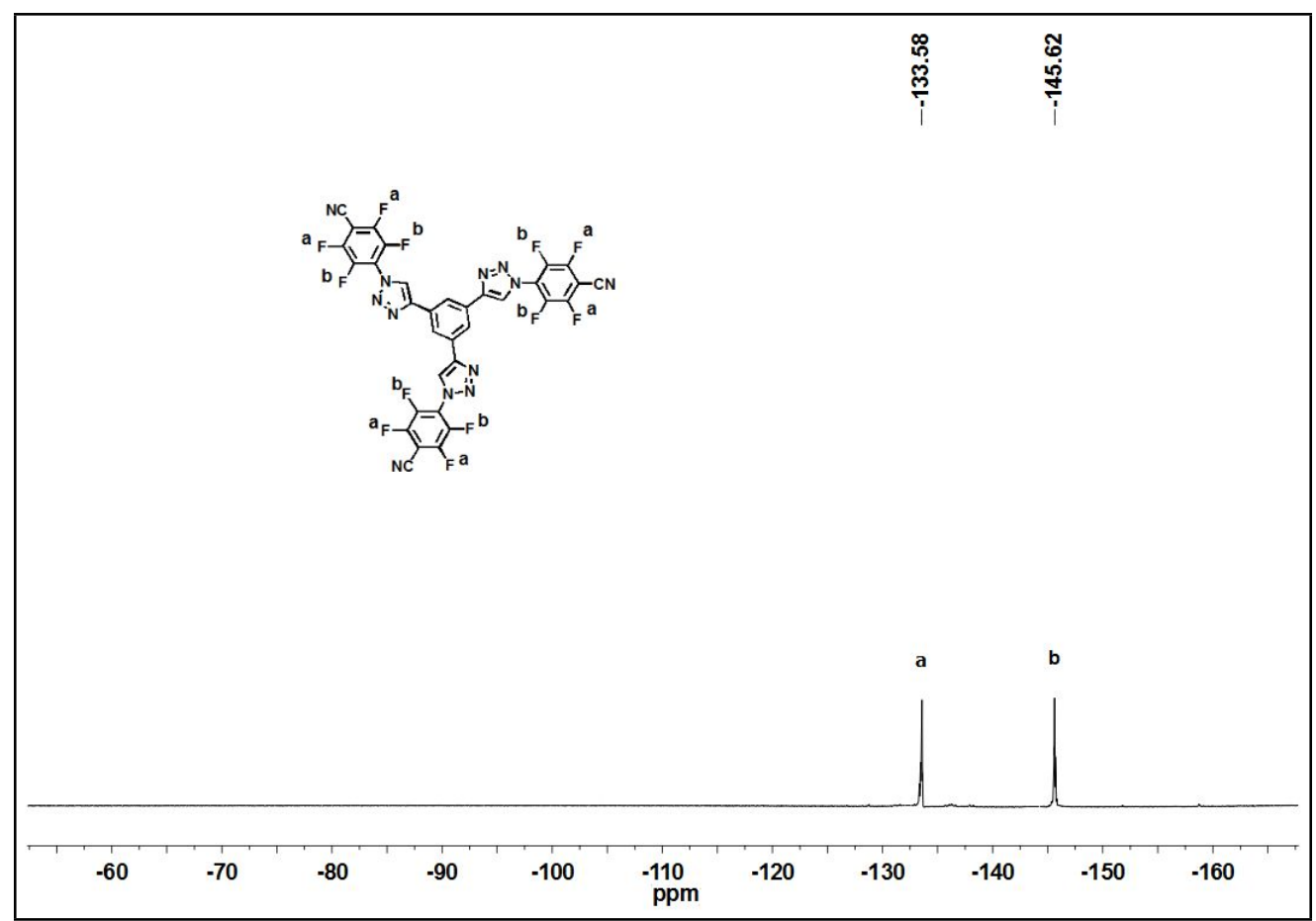

Figure S4: ${ }^{19} \mathrm{~F}$ NMR of Tz-PFCN building block

\section{Section D. Elemental Analysis:}

Table S1. Elemental analysis of Tz- $d f$-CTF materials from combustion process

\begin{tabular}{|c|c|c|c|c|c|}
\hline \multirow{2}{*}{ Sample } & \multicolumn{2}{|c|}{ Elemental Analysis (Experimental) } & \multirow{2}{*}{ C/N } & \multirow{2}{*}{ C/H } \\
\cline { 2 - 4 } & $\mathbf{C}$ & $\mathbf{N}$ & $\mathbf{H}$ & & \\
\hline Tz- $d f$-CTF400 & 49.39 & 9.63 & 1.74 & 5.12 & 28.38 \\
\hline Tz- $d f$-CTF500 & 47.90 & 7.97 & 2.04 & 6.01 & 23.48 \\
\hline Tz- $d f$-CTF600 & 48.39 & 6.15 & 2.25 & 7.86 & 21.50 \\
\hline Calc. Tz-PFCN & 49.64 & 21.05 & 0.76 & 2.35 & 65.31 \\
\hline
\end{tabular}

Section E. XPS Survey Scan of Tz-df-CTFs:

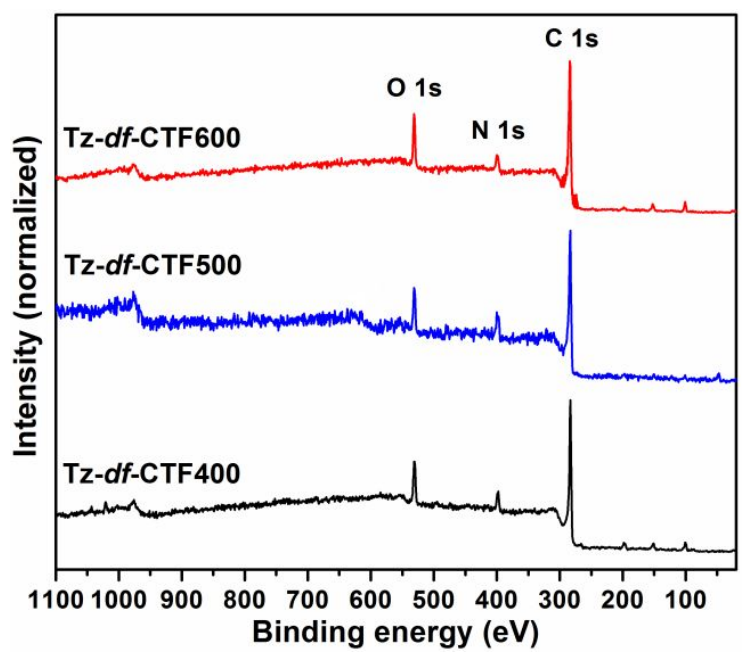

Figure S5: XPS survey spectra Tz- $d f$-CTFs 
Section F. Wide-Angle Powder XRD Analysis of Tz-df-CTFs:

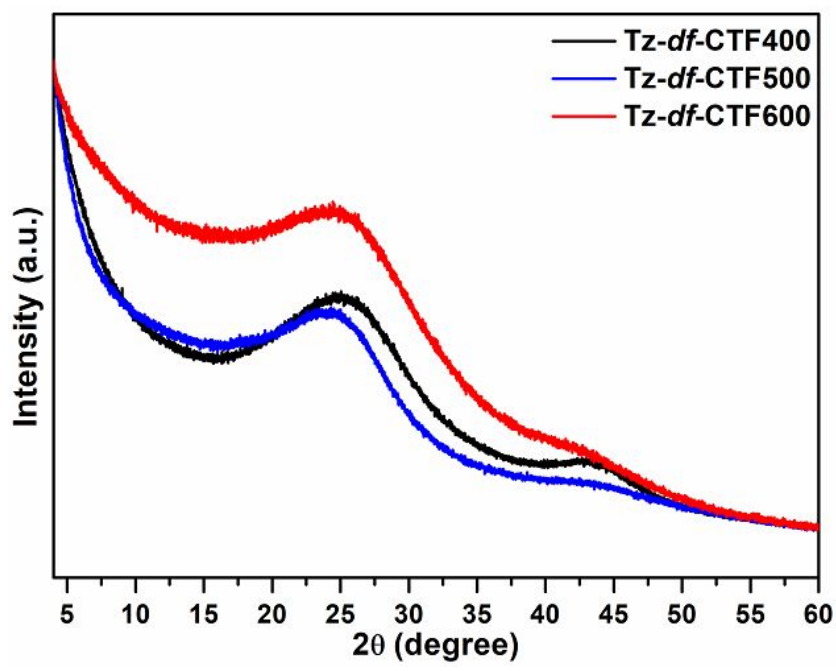

Figure S6. Powder X-ray diffraction spectra of Tz-df-CTFs.

Section G. FE-SEM and HRTEM Analysis of Tz-df-CTFs:

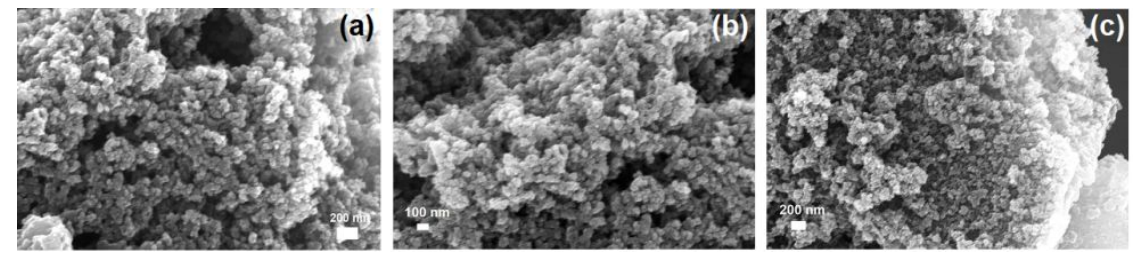

Figure S7. FE-SEM images of (a) Tz- $d f-C T F 400$, (b) Tz- $d f-C T F 500$, and (c) Tz- $d f-C T F 600$.
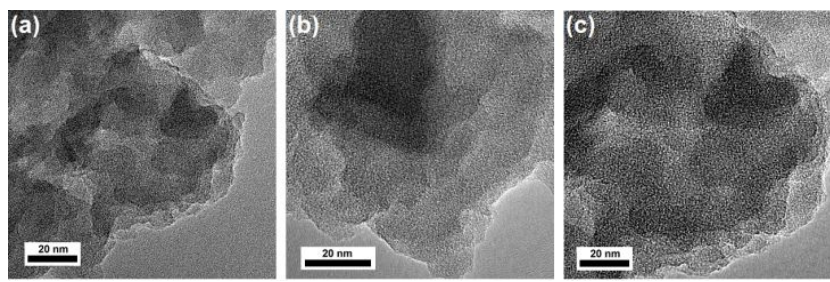

Figure S8. HR-TEM image (a) Tz- $d f$-CTF400, (b) Tz- $d f$-CTF500, and (c) Tz- $d f$-CTF600.

Section H. Pore Size Distribution Curves Derived from $\mathrm{CO}_{2}$ Isotherm:

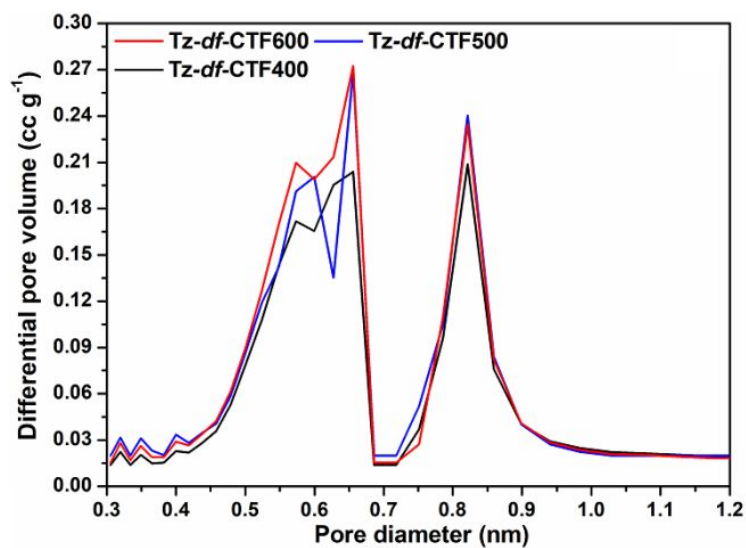

Figure S9. Pore size distribution curve of Tz- $d f-\mathrm{CTF}$ s derived from $\mathrm{CO}_{2}$ adsorption isotherms $(273 \mathrm{~K})$ using NL-DFT method. 


\section{Section I. Fitting of Unary Isotherm Data:}

The unary isotherm data for $\mathrm{CO}_{2}, \mathrm{CH}_{4}$, and $\mathrm{N}_{2}$, measured at $273 \mathrm{~K}$ and $298 \mathrm{~K}$ in Tz-df-CTFs, were fitted with excellent accuracy with the single-site Langmuir isotherm model

$$
q=q_{\text {sat }} \frac{b p}{1+b p}
$$

with $T$-dependent parameter $b$

$$
b=b_{0} \exp \left(\frac{E}{R T}\right)
$$

The fitted parameter values are presented in Table S2,

Table S3, and Table S4 derived from unary gas isotherm data of all Tz- $d f$-CTFs measured at $273 \mathrm{~K}$ and $298 \mathrm{~K}$.

Table S2. Langmuir fit parameter values for $\mathrm{CO}_{2}, \mathrm{CH}_{4}$, and $\mathrm{N}_{2}$ in Tz-df-CTF400.

\begin{tabular}{|c|c|c|c|}
\hline $\mathbf{G a s}$ & $\boldsymbol{q}_{\text {sat }}\left(\mathbf{m o l} \mathbf{~ k g}^{-1}\right)$ & $\boldsymbol{b}_{\mathbf{0}}\left(\mathbf{P a}^{-\mathbf{1}}\right)$ & $\boldsymbol{E}\left(\mathbf{k J} \mathbf{~ m o l}^{-\mathbf{1}}\right)$ \\
\hline $\mathrm{CO}_{2}$ & 8.5 & $8.42 \mathrm{E}-10$ & 23.4 \\
\hline $\mathrm{CH}_{4}$ & 5.4 & $4.04 \mathrm{E}-11$ & 27.4 \\
\hline $\mathrm{N}_{2}$ & 12.6 & $3.32 \mathrm{E}-11$ & 23.2 \\
\hline
\end{tabular}

Table S3. Langmuir fit parameter values for $\mathrm{CO}_{2}, \mathrm{CH}_{4}$, and $\mathrm{N}_{2}$ in Tz- $d f$-CTF500.

\begin{tabular}{|c|c|c|c|}
\hline $\mathbf{G a s}$ & $\boldsymbol{q}_{\text {sat }}\left(\mathbf{m o l} \mathbf{~ k g}^{-\mathbf{1}}\right)$ & $\boldsymbol{b}_{\mathbf{0}}\left(\mathbf{P a}^{\mathbf{- 1}}\right)$ & $\boldsymbol{E}\left(\mathbf{k J} \mathbf{~ m o l}^{-\mathbf{1}}\right)$ \\
\hline $\mathrm{CO}_{2}$ & 11 & $2.90 \mathrm{E}-09$ & 19.7 \\
\hline $\mathrm{CH}_{4}$ & 11 & $1.51 \mathrm{E}-10$ & 22.5 \\
\hline $\mathrm{N}_{2}$ & 15 & $3.10 \mathrm{E}-12$ & 28.6 \\
\hline
\end{tabular}

Table S4. Langmuir fit parameter for $\mathrm{CO}_{2}, \mathrm{CH}_{4}$, and $\mathrm{N}_{2}$ in Tz-df-CTF600.

\begin{tabular}{|c|c|c|c|}
\hline $\mathbf{G a s}$ & $\boldsymbol{q}_{\text {sat }}\left(\mathbf{m o l ~ k g} \mathbf{~}^{-1}\right)$ & $\boldsymbol{b}_{\mathbf{0}}\left(\mathbf{P a}^{-\mathbf{1}}\right)$ & $\boldsymbol{E}\left(\mathbf{k J} \mathbf{~ m o l}^{-\mathbf{1}}\right)$ \\
\hline $\mathrm{CO}_{2}$ & 11.6 & $2.48 \mathrm{E}-09$ & 20 \\
\hline $\mathrm{CH}_{4}$ & 7.1 & $1.57 \mathrm{E}-10$ & 24 \\
\hline $\mathrm{N}_{2}$ & 15 & $1.01 \mathrm{E}-11$ & 26 \\
\hline
\end{tabular}

\section{Section J. Isosteric Heat of Adsorption:}

The isosteric heat of adsorption, $Q_{\text {st }}$, defined as

$Q_{s t}=R T^{2}\left(\frac{\partial \ln p}{\partial T}\right)_{q}$

were determined from the pure component isotherm fits at $298 \mathrm{~K}$ and $273 \mathrm{~K}$ using the Clausius-Clapeyron equation. 


\section{Section K. IAST Calculation of Component Uptakes and Adsorption Selectivities:}

The IAST calculations were performed for binary $15 / 85 \mathrm{CO}_{2} / \mathrm{N}_{2}$ and $50 / 50 \mathrm{CO}_{2} / \mathrm{CH}_{4}$ gas mixtures at $298 \mathrm{~K}$. The adsorption selectivity was calculated using equation

$$
S_{a d s}=\frac{q_{A} / q_{B}}{y_{A} / y_{B}}
$$

where the $q_{\mathrm{A}}$, and $q_{\mathrm{B}}$ represent the molar loadings of Tz- $d f$-CTFs that is in equilibrium with a bulk fluid mixture with mole fractions $y_{\mathrm{A}}$, and $y_{\mathrm{B}}=1-y_{\mathrm{A}}$. The molar loadings, also called gravimetric uptake capacities, are expressed in $\mathrm{mol} \mathrm{kg}{ }^{-1}$. For $15 / 85 \mathrm{CO}_{2} / \mathrm{N}_{2}$ separations, the mole fractions $y_{\mathrm{A}}=0.15$ and $y_{\mathrm{B}}=1-y_{\mathrm{A}}=0.85$. For $50 / 50 \mathrm{CO}_{2} / \mathrm{CH}_{4}$ separations, the mole fractions $y_{\mathrm{A}}=0.5$ and $y_{\mathrm{B}}=1-y_{\mathrm{A}}=0.5$.
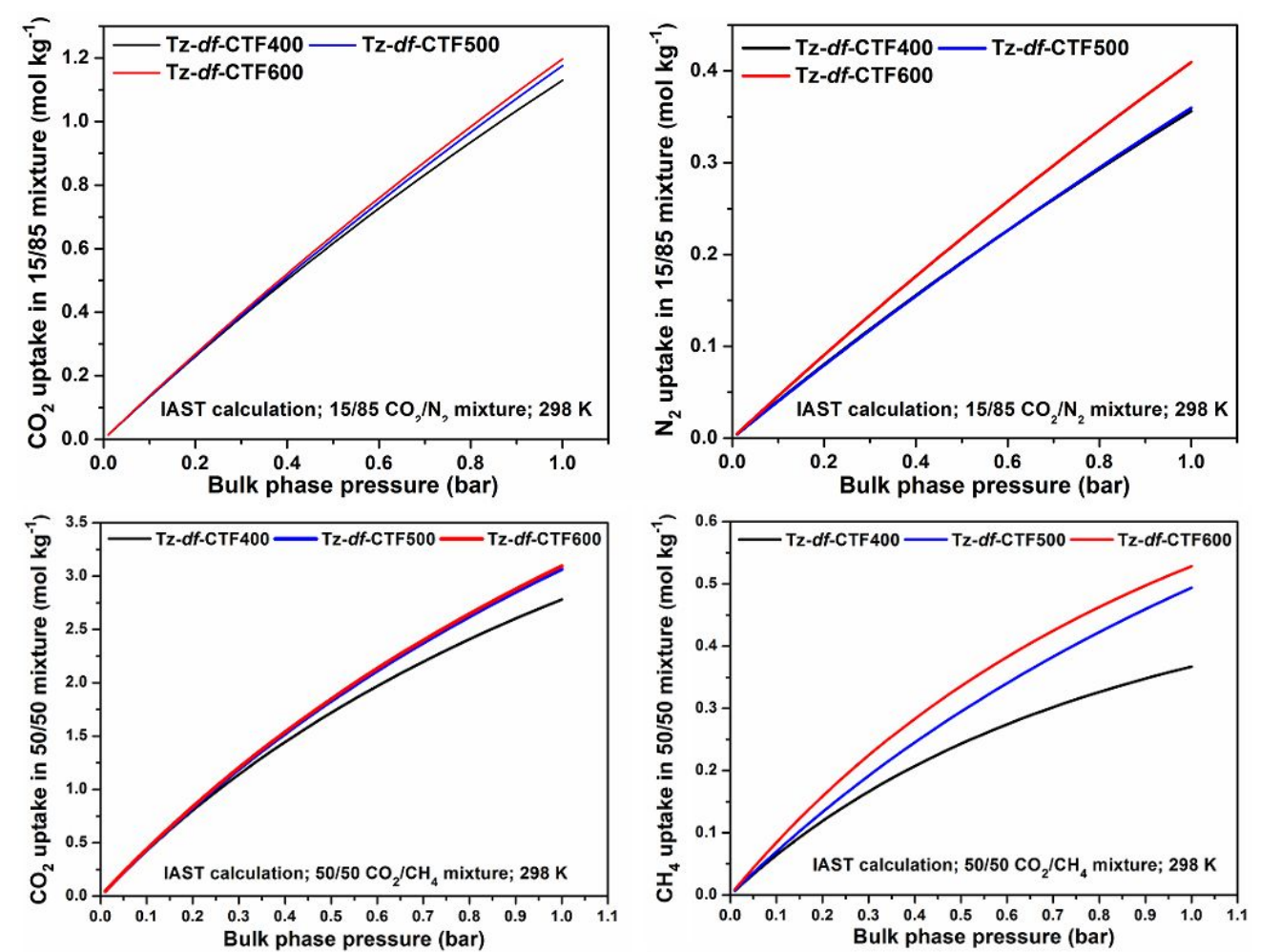

Figure S10. Calculations using Ideal Adsorbed Solution Theory (IAST) for uptakes of $\mathrm{CO}_{2}$, $\mathrm{CH}_{4}$ and $\mathrm{N}_{2}$, expressed as moles per $\mathrm{kg}$ of adsorbent, in equilibrium with binary $\mathrm{CO}_{2} / \mathrm{N}_{2}$ as well as $\mathrm{CO}_{2} / \mathrm{CH}_{4}$ gas mixture maintained at isothermal condition at $298 \mathrm{~K}$. In these calculations the partial pressures of $\mathrm{CO}_{2}$ and $\mathrm{N}_{2}$ for $\mathrm{CO}_{2} / \mathrm{N}_{2}$ mixture are taken to be $p_{1} / p_{2}=$ $15 / 85$ and $p_{1} / p_{2}=50 / 50$ for $\mathrm{CO}_{2} / \mathrm{CH}_{4}$ mixture 

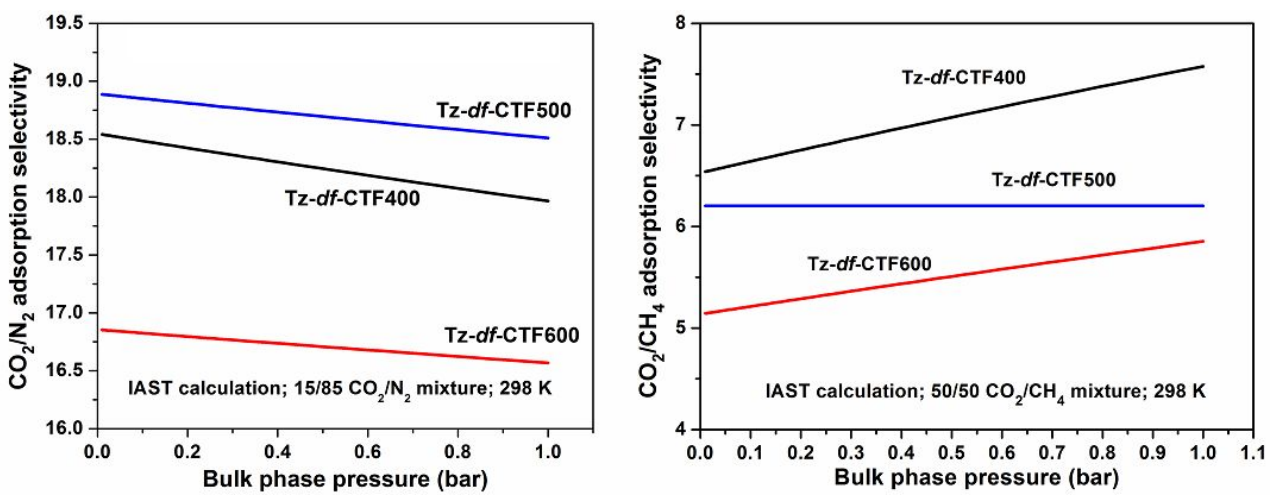

Figure S11. Calculations using Ideal Adsorbed Solution Theory (IAST) for $\mathrm{CO}_{2} / \mathrm{N}_{2}$ and $\mathrm{CO}_{2} / \mathrm{CH}_{4}$ adsorption selectivity for a binary $\mathrm{CO}_{2} / \mathrm{N}_{2}$ and $\mathrm{CO}_{2} / \mathrm{CH}_{4}$ gas mixture and maintained at isothermal condition at $298 \mathrm{~K}$. In these calculations the partial pressures of $\mathrm{CO}_{2}$ and $\mathrm{N}_{2}$ are taken to be $p_{1} / p_{2}=15 / 85$ and for $\mathrm{CO}_{2}$ and $\mathrm{CH}_{4}$ are taken to be $p_{1} / p_{2}=50 / 50$

\section{Section L. Transient Breakthrough of mixtures in Fixed Bed Adsorbers:}

The performance of industrial fixed bed adsorbers is dictated by a combination of adsorption selectivity and uptake capacity. Transient breakthrough simulations were carried out for binary $15 / 85 \mathrm{CO}_{2} / \mathrm{N}_{2}$ and $50 / 50 \mathrm{CO}_{2} / \mathrm{CH}_{4}$ gas mixtures operating at a total pressure of 100 $\mathrm{kPa}$ and $298 \mathrm{~K}$, using the simulation methodology described in earlier publications as stated in main text. In a typical breakthrough simulation, the following parameter values were used: length of packed bed, $L=0.3 \mathrm{~m}$; voidage of packed bed, $\varepsilon=0.4$; superficial gas velocity at inlet, $u=0.04 \mathrm{~m} / \mathrm{s}$. The transient breakthrough simulation results are presented in terms of a dimensionless time, $\tau=\frac{t u}{L \varepsilon}$. The $y$-axis is \% of component in the gas mixture exiting the adsorber. The partial pressures of $\mathrm{CO}_{2}$ and $\mathrm{N}_{2}$ in $15 / 85 \mathrm{CO}_{2} / \mathrm{N}_{2}$ binary gas mixture at the feed gas inlet are $15 \mathrm{kPa}$, and $85 \mathrm{kPa}$, respectively. Similarly, the partial pressures of $\mathrm{CO}_{2}$ and $\mathrm{CH}_{4}$ in $50 / 50 \mathrm{CO}_{2} / \mathrm{CH}_{4}$ binary mixture at the feed gas inlet are $50 \mathrm{kPa}$, and $50 \mathrm{kPa}$, respectively.

\section{Section M. Henry Selectivity Studies:}

Table S5. Initial Slopes for individual isotherm and Henry Selectivity of Tz- $d f$-CTFs.

\begin{tabular}{|c|c|c|c|c|c|c|c|c|c|}
\hline \multirow{3}{*}{ Temp. } & \multicolumn{9}{|c|}{ Initial slope } \\
\hline & \multicolumn{3}{|c|}{ Tz-df-CTF400 } & \multicolumn{3}{|c|}{ Tz-df-CTF500 } & \multicolumn{3}{|c|}{ Tz-df-CTF600 } \\
\hline & $\mathrm{CO}_{2}$ & $\mathrm{CH}_{4}$ & $\mathbf{N}_{2}$ & $\mathrm{CO}_{2}$ & $\mathrm{CH}_{4}$ & $\mathbf{N}_{2}$ & $\mathrm{CO}_{2}$ & $\mathrm{CH}_{4}$ & $\mathbf{N}_{2}$ \\
\hline $273 K$ & 19.331 & 4.072 & 1.139 & 20.986 & 3.439 & 1.444 & 21.594 & 4.301 & 1.418 \\
\hline $298 K$ & 11.468 & 1.425 & 0.504 & 11.260 & 1.562 & 0.524 & 11.142 & 1.941 & 0.528 \\
\hline \multirow{3}{*}{ Temp. } & \multicolumn{9}{|c|}{ Henry selectivity } \\
\hline & \multicolumn{3}{|c|}{ Tz-df-CTF400 } & \multicolumn{3}{|c|}{ Tz-df-CTF500 } & \multicolumn{3}{|c|}{ Tz-df-CTF600 } \\
\hline & \multicolumn{2}{|c|}{$\mathrm{CO}_{2} / \mathrm{CH}_{4}$} & $\mathrm{CO}_{2} / \mathrm{N}_{2}$ & \multicolumn{2}{|c|}{$\mathrm{CO}_{2} / \mathrm{CH}_{4}$} & $\mathrm{CO}_{2} / \mathrm{N}_{2}$ & \multicolumn{2}{|c|}{$\mathrm{CO}_{2} / \mathrm{CH}_{4}$} & $\mathrm{CO}_{2} / \mathrm{N}_{2}$ \\
\hline $273 K$ & 4.72 & & 16.971 & 6.102 & & 4.533 & 5.02 & & 15.228 \\
\hline
\end{tabular}




\begin{tabular}{|l|l|l|l|l|l|l|}
\hline 298K & 8.047 & 22.754 & 7.208 & 21.488 & 5.740 & 21.102 \\
\hline
\end{tabular}

Section N. Henry Plot for $\mathrm{CO}_{2}, \mathrm{~N}_{2}$, and $\mathrm{CH}_{4}$ :
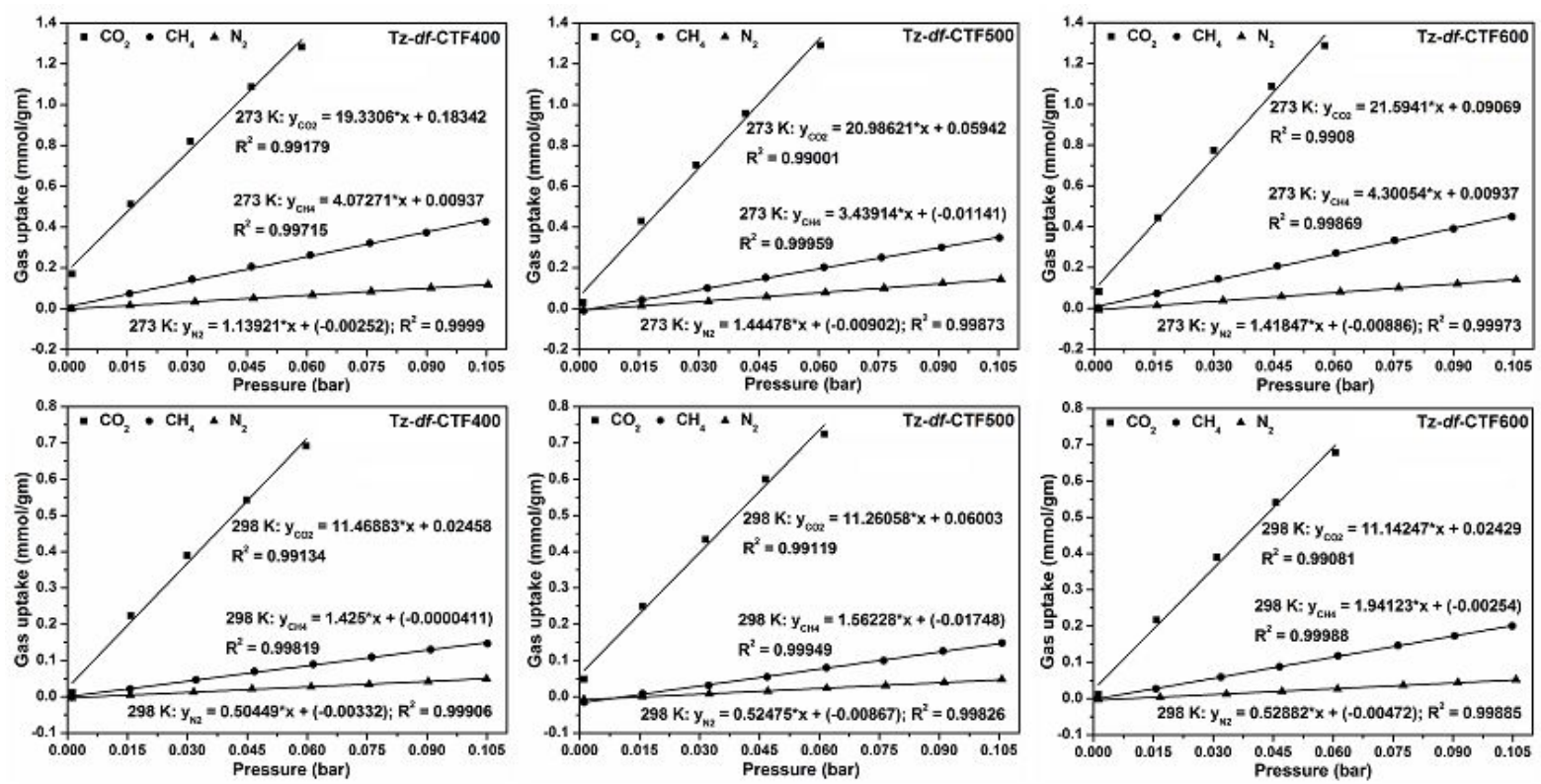

Figure S12. Henry plots of all Tz- $d f-\mathrm{CTF}$ materials as derived from $\mathrm{CO}_{2}, \mathrm{~N}_{2}$, and $\mathrm{CH}_{4}$ isotherms at $\mathrm{T}=273$ and $298 \mathrm{~K}$.

\section{Section O. Productivity for $\mathrm{CO}_{2} / \mathrm{CH}_{4}$ Separation:}

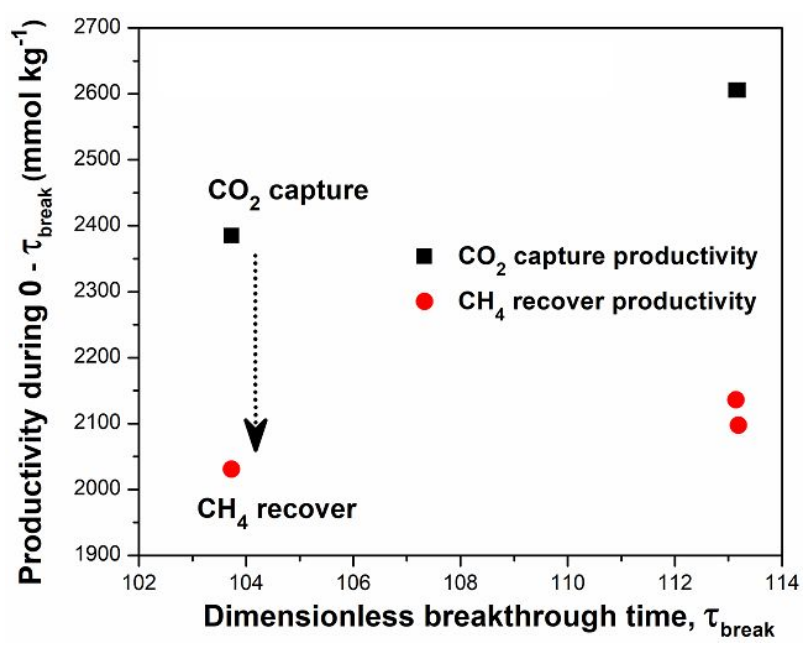

Figure S13. Comparison of $\mathrm{CO}_{2}$ capture and $\mathrm{CH}_{4}$ recovery productivity at $298 \mathrm{~K}$.

\section{Section P. Comparison Tables of Textural and Gas Uptake Properties:}

Table S6. Comparison of surface area, $\mathrm{CO}_{2}$ uptake ( $>4 \mathrm{mmol} \mathrm{g} \mathrm{g}^{-1}$ at $\left.273 \mathrm{~K}\right), Q_{\text {st }}$, selectivity $\left(\mathrm{CO}_{2} / \mathrm{N}_{2}\right)$ and $\mathrm{H}_{2}$ uptake of Tz-df-CTF materials with notable POP based adsorbents at 1 bar.

\begin{tabular}{|c|c|c|c|c|c|c|c|c|}
\hline \multirow{2}{*}{$\begin{array}{l}\text { POP-based } \\
\text { Adsorbents }\end{array}$} & \multirow{2}{*}{$\begin{array}{c}\begin{array}{c}\mathbf{S}_{\mathrm{BET}} \\
\left(\mathbf{m}^{2} \mathbf{g}^{-1}\right)\end{array} \\
77 \mathrm{~K}\end{array}$} & \multicolumn{2}{|c|}{$\begin{array}{l}\mathrm{CO}_{2} \text { Uptake } \\
{\left[\mathrm{mmol} \mathrm{g}^{-1}\right]}\end{array}$} & \multirow{2}{*}{$\underset{\left[\mathrm{KJ} \mathrm{mol}^{-1}\right]}{Q_{\text {st }}}$} & \multicolumn{2}{|c|}{$\begin{array}{c}\left(\mathrm{CO}_{2} / \mathrm{N}_{2}\right) \\
\text { Selectivity } \\
\text { at } 298 \mathrm{~K}\end{array}$} & $\begin{array}{c}\mathrm{H}_{2} \text { Uptake } \\
{[\mathbf{w t} \%]}\end{array}$ & \multirow[t]{2}{*}{ Ref. } \\
\hline & & $273 \mathrm{~K}$ & $298 \mathrm{~K}$ & & Henry & IAST & $77 \mathrm{~K}$ & \\
\hline
\end{tabular}




\begin{tabular}{|c|c|c|c|c|c|c|c|c|}
\hline Tz-df-CTF600 & 2105 & 7.65 & 5.08 & 20.0 & 21.1 & 16.8 & 2.91 & Our work \\
\hline Tz-df-CTF500 & 1878 & 7.41 & 4.86 & 19.7 & 21.5 & 18.9 & 2.76 & Our work \\
\hline Tz-df-CTF400 & 1550 & 6.42 & 4.32 & 23.4 & 22.7 & 18.5 & 2.51 & Our work \\
\hline CQN-1e & 1931 & 6.58 & 4.39 & 41.7 & - & 44.6 & - & \multirow[b]{2}{*}{95} \\
\hline CQN-1g & 1870 & 7.16 & 4.57 & 40.6 & - & 42.7 & - & \\
\hline NRPP-1 & 1579 & 6.10 & 2.22 & 28 & 21 & 21 & - & \multirow{2}{*}{94} \\
\hline NRPP-2 & 1028 & 7.06 & 3.71 & 29.1 & 24 & 36 & - & \\
\hline CPOP-1 & 2220 & 4.82 & - & 27 & - & $25^{a}$ & 2.80 & 98 \\
\hline PPF-1 & 1740 & 6.12 & - & 25.6 & - & $14.5^{a}$ & 2.75 & \multirow[b]{2}{*}{89} \\
\hline PPF-2 & 1470 & 5.54 & - & 29.2 & - & $15.4^{a}$ & 2.28 & \\
\hline NPOF-1-NH ${ }_{2}$ & 1535 & 5.84 & 3.77 & 32.1 & - & 25 & - & S3 \\
\hline TPMTP & 890 & 5.80 & 3.60 & 28.3 & $63^{a}$ & $61^{a}$ & - & 36 \\
\hline $\mathrm{P}-\mathrm{PCz}$ & 1647 & 5.57 & 2.97 & 30.9 & $32^{a}$ & $42^{a}$ & - & 90 \\
\hline ALP-1 & 1235 & 5.37 & 3.25 & 29.2 & 27 & 28 & 2.19 & \multirow[b]{2}{*}{91} \\
\hline ALP-2 & 1065 & 4.80 & 2.46 & 27.9 & 27 & 26 & 1.74 & \\
\hline BILP-4 & 1135 & 5.34 & 3.59 & 28.7 & 32 & - & 2.30 & \multirow{4}{*}{ S4 } \\
\hline BILP-3 & 1306 & 5.11 & 3.30 & 28.6 & 31 & - & 2.10 & \\
\hline BILP-6 & 1261 & 4.80 & 2.75 & 28.4 & 39 & - & 2.20 & \\
\hline BILP-7 & 1122 & 4.39 & 2.77 & 27.8 & 34 & - & 1.80 & \\
\hline TBILP-2 & 1080 & 5.59 & 3.32 & 29.0 & 40 & 43 & - & S5 \\
\hline TpPaCOF(MW) & 724.6 & 5.10 & 2.00 & 34.1 & - & $32^{a}$ & - & S6 \\
\hline COF-JLU-2 & 410 & 4.93 & - & 31 & 77 & - & 1.60 & S7 \\
\hline N-PEINK & 1836 & 4.86 & - & 31.3 & $97^{a}$ & - & 2.67 & S8 \\
\hline Cz-POF-3 & 1927 & 4.77 & - & 27.8 & 23 & $20^{a}$ & 2.07 & \multirow{2}{*}{ S9 } \\
\hline Cz-POF-1 & 2065 & 4.59 & - & 25.3 & 19 & $17^{a}$ & 2.24 & \\
\hline $\mathrm{Zn} @ \mathrm{AB}-\mathrm{COF}$ & 1120 & 4.68 & 1.79 & 31.8 & 48 & 55 & - & \multirow{2}{*}{ S10 } \\
\hline Li@AB-COF & 988 & 4.33 & 1.43 & 32.2 & 13 & 16 & - & \\
\hline MCTP-1 & 1452 & 4.64 & 2.70 & 40 & 15.4 & - & - & S11 \\
\hline PP-N-25 & 1257 & 4.60 & - & $\sim 35$ & - & - & - & $\mathrm{S} 12$ \\
\hline HEX-COF-1 & 1200 & 4.55 & - & 42 & - & - & - & $\mathrm{S} 13$ \\
\hline APOP-3 & 1402 & 4.54 & 2.59 & 27.5 & - & 26.0 & 1.80 & \multirow{2}{*}{ S14 } \\
\hline APOP-1 & 1298 & 4.26 & 2.69 & 26.6 & - & 20.2 & 1.71 & \\
\hline SIOC-COF-5 & 707 & 4.50 & - & - & - & - & 1.66 & S15 \\
\hline SNU-C1-sca & 830 & 4.38 & 3.14 & 31.2 & - & 88 & - & S16 \\
\hline CZ@PON & 592 & 4.31 & 1.67 & 42.01 & 107 & - & 1.65 & \multirow{2}{*}{ S17 } \\
\hline BCzMB@PON & 865 & 4.02 & 1.43 & 31.96 & 58 & - & 1.57 & \\
\hline Fe-POP-1 & 875 & 4.31 & - & - & - & - & - & S18 \\
\hline HC-PCz-8 & 1688 & 4.30 & 3.50 & 20.4 & 17 & - & - & S19 \\
\hline
\end{tabular}




\begin{tabular}{|c|c|c|c|c|c|c|c|c|}
\hline BILP-1 & 1172 & 4.27 & 2.98 & 26.5 & 36 & - & 1.90 & S20 \\
\hline POF1B & 917 & 4.23 & 2.16 & - & - & - & 1.25 & S21 \\
\hline PPN-6-SO 3 Li & 1186 & 4.20 & - & 35.7 & - & $17^{b}$ & - & 35 \\
\hline STP-2 & 1990 & 4.14 & - & - & - & - & 1.93 & S22 \\
\hline CPOP-9 & 2440 & 4.14 & 2.23 & 24.5 & - & - & 2.44 & 99 \\
\hline TSP-2 & 913 & 4.10 & 2.6 & 30.8 & 38 & 25 & - & S23 \\
\hline TB-MOP & 694 & 4.05 & 2.57 & 24.5 & 50.6 & - & - & S24 \\
\hline BILP-10 & 787 & 4.02 & $\sim 2.5$ & 38.2 & 59 & - & 1.60 & S25 \\
\hline PAN-2 & 1242 & 4.00 & 2.89 & 39.3 & $104^{a}$ & $50^{a}$ & - & 100 \\
\hline
\end{tabular}

${ }^{a}$ measured at $273 \mathrm{~K} .{ }^{b}$ measured at $295 \mathrm{~K}$.

Table S7. Comparison of structural parameters, $\mathrm{CO}_{2}\left(>4 \mathrm{mmol} \mathrm{g}{ }^{-1}\right.$ at $\left.273 \mathrm{~K}\right)$ and $\mathrm{H}_{2}$ uptakes, and $\mathrm{CO}_{2} / \mathrm{N}_{2}$ selectivity of Tz-df-CTF materials with other most representative related CTFbased adsorbents.

\begin{tabular}{|c|c|c|c|c|c|c|c|c|}
\hline \multirow[t]{2}{*}{$\begin{array}{c}\text { Only CTF-based } \\
\text { adsorbents }\end{array}$} & \multirow{2}{*}{$\begin{array}{c}\begin{array}{c}\mathbf{S}_{\mathrm{BET}} \\
\left(\mathbf{m}^{\mathbf{2}} \mathbf{g}^{-1}\right)\end{array} \\
77 \mathrm{~K}\end{array}$} & \multicolumn{2}{|c|}{$\begin{array}{c}\mathrm{CO}_{2} \text { Uptake } \\
{\left[\mathrm{mmol} \mathrm{g}^{-1}\right]}\end{array}$} & \multirow[t]{2}{*}{$\underset{\left[\mathrm{KJ} \mathrm{mol}^{-1}\right]}{Q_{\text {st }}}$} & \multicolumn{2}{|c|}{$\begin{array}{c}\left(\mathrm{CO}_{2} / \mathrm{N}_{2}\right) \\
\text { Selectivity } \\
\text { at } 298 \mathrm{~K}\end{array}$} & \multirow{2}{*}{$\begin{array}{c}\begin{array}{c}\mathbf{H}_{2} \\
\text { Uptake } \\
\text { [wt\%] }\end{array} \\
77 \mathrm{~K}\end{array}$} & \multirow[t]{2}{*}{ Ref. } \\
\hline & & $273 \mathrm{~K}$ & $298 \mathrm{~K}$ & & Henry & IAST & & \\
\hline Tz- $d f-\mathrm{CTF} 600$ & 2105 & 7.65 & 5.08 & 20.0 & 21.1 & 16.8 & 2.91 & Our work \\
\hline $\mathrm{Tz}-d f-\mathrm{CTF} 500$ & 1878 & 7.41 & 4.86 & 19.7 & 21.5 & 18.9 & 2.76 & Our work \\
\hline Tz-df-CTF400 & 1550 & 6.42 & 4.32 & 23.4 & 22.7 & 18.5 & 2.51 & Our work \\
\hline HAT-CTF $450 / 600$ & 1090 & 6.3 & $4.80^{a}$ & 27.1 & $126^{a}$ & - & - & \multirow{3}{*}{32} \\
\hline HAT-CTF-600 & 899 & 5.1 & - & - & - & - & - & \\
\hline HAT-CTF-450 & 756 & 4.4 & - & - & - & - & - & \\
\hline caCTF-1-700 & 2367 & 6.00 & 3.55 & 30.6 & - & - & 2.46 & \multirow{2}{*}{53} \\
\hline caCTF-2-700 & 1866 & 5.10 & 3.10 & 31.9 & - & - & 1.89 & \\
\hline F-DCBP-CTF-1 & 2437 & 5.98 & 3.82 & 33.1 & 31 & - & 1.77 & \multirow{2}{*}{55} \\
\hline F-DCBP-CTF-2 & 2036 & 5.23 & 3.16 & - & 22 & - & - & \\
\hline CTF-py ${ }^{\mathrm{HT}}$ & 3040 & 5.97 & 4.22 & 27.1 & 29 & - & 2.63 & \multirow{4}{*}{96} \\
\hline CTF-py & 1239 & 5.08 & 3.79 & 35.1 & 45 & - & 1.63 & \\
\hline CTF-ph & 1991 & 4.54 & 3.05 & 33.2 & 20 & - & 1.87 & \\
\hline CTF-ph ${ }^{\mathrm{HT}}$ & 2675 & 4.17 & 2.69 & 25.4 & 11 & - & 2.46 & \\
\hline bipy-CTF500 & 1548 & 5.34 & 3.07 & 34.2 & 61 & 42 & 1.63 & \multirow{6}{*}{56} \\
\hline bipy-CTF600 & 2479 & 5.58 & 2.95 & 34.4 & 37 & 24 & 2.10 & \\
\hline lut-CTF350 & 635 & 4.06 & 2.41 & 37.4 & 76 & 66 & 1.22 & \\
\hline lut-CTF400 & 968 & 4.55 & 2.72 & 37.5 & 63 & 53 & 1.36 & \\
\hline lut-CTF500 & 1680 & 5.04 & 2.58 & 38.2 & 27 & 27 & 1.60 & \\
\hline lut-CTF600 & 2815 & 4.99 & 2.52 & 33.3 & 26 & 23 & 2.00 & \\
\hline FCTF-1-600 & 1535 & 5.53 & 3.41 & - & - & 19 & - & 110 \\
\hline
\end{tabular}




\begin{tabular}{|c|c|c|c|c|c|c|c|c|}
\hline FCTF-1 & 662 & 4.67 & 3.21 & 35 & - & 31 & - & \\
\hline$p \mathrm{CTF}-1$ & 2034 & 4.97 & - & 25.9 & - & & 1.75 & S26 \\
\hline TPC-1 & 1940 & 4.90 & - & 39 & $38^{b}$ & & - & 63 \\
\hline MM2 & 1360 & 4.77 & - & 32 & $23^{b}$ & $44^{b}$ & 1.74 & 68 \\
\hline PCTF-4 & 1404 & 4.65 & 2.86 & $\sim 31$ & $56^{b}$ & - & 1.30 & 39 \\
\hline$f l-\mathrm{CTF}-350$ & 1235 & 4.28 & 2.29 & 32.7 & 27 & 23 & - & 27 \\
\hline$f l-\mathrm{CTF}-400$ & 2862 & 4.13 & 1.97 & 30.7 & 15 & 16 & 1.95 & ונד \\
\hline CTF-0 & 2011 & 4.22 & - & - & - & - & - & 38 \\
\hline P6M & 947 & 4.17 & - & - & - & $14.2^{b}$ & & $\mathrm{~S} 27$ \\
\hline PCTP-1 & 1200 & 4.91 & 3.24 & 44.5 & 46.1 & - & - & $\mathrm{S} 28$ \\
\hline CTF-BI-4 & 1025 & 4.85 & - & \multirow{5}{*}{$34.3-31.7$} & $44.0^{b}$ & - & - & \multirow{5}{*}{$\mathrm{S} 29$} \\
\hline CTF-BI-5 & 836 & 4.49 & - & & $35.6^{b}$ & - & - & \\
\hline CTF-BI-9 & 885 & 4.29 & - & & $67.4^{b}$ & - & - & \\
\hline CTF-BI-10 & 1099 & 4.45 & - & & $31.3^{b}$ & - & - & \\
\hline CTF-BI-11 & 1549 & 4.93 & - & & $34.3^{b}$ & - & - & \\
\hline MCTP-1 & 1452 & 4.64 & 2.69 & 40.0 & 15.4 & - & - & $\mathrm{S} 11$ \\
\hline CTF-TPC & 1668 & 4.25 & - & 32 & $20.0^{b}$ & $30.0^{b}$ & 1.75 & S30 \\
\hline
\end{tabular}

${ }^{a}$ measured at $297 \mathrm{~K},{ }^{b}$ measured at $273 \mathrm{~K}$.

Table S8. Comparison of surface area, $\mathrm{CH}_{4}$ uptake $\left(>2 \mathrm{wt} \%\right.$ at $273 \mathrm{~K}$ and 1 bar) and $Q_{\mathrm{st}}$ of other high-performing POP and CTF based adsorbents.

\begin{tabular}{|c|c|c|c|c|c|}
\hline \multirow{2}{*}{$\begin{array}{l}\text { POP \& CTF-based } \\
\text { adsorbents }\end{array}$} & \multirow{2}{*}{$\frac{\mathrm{S}_{\text {BET }}\left(\mathrm{m}^{2} \mathrm{~g}^{-1}\right)}{77 \mathrm{~K}}$} & \multicolumn{2}{|c|}{$\mathrm{CH}_{4}$ Uptake [wt\%] } & \multirow{2}{*}{$\begin{array}{c}Q_{\text {st }} \\
{\left[\mathrm{KJ} \mathrm{mol}^{-1}\right]}\end{array}$} & \multirow{2}{*}{ Ref. } \\
\hline & & $273 \mathrm{~K}$ & $298 \mathrm{~K}$ & & \\
\hline TPC-1 & 1940 & 6.2 & - & - & 63 \\
\hline Tz-df-CTF600 & 2105 & 4.41 & 2.20 & 24.0 & Our work \\
\hline Tz-df-CTF500 & 1878 & 4.13 & 2.08 & 22.5 & Our work \\
\hline Tz- $d f-\mathrm{CTF} 400$ & 1550 & 3.64 & 1.73 & 27.4 & Our work \\
\hline PPOP-1 & 720 & 4.01 & - & - & \multirow{3}{*}{$\mathrm{S} 31$} \\
\hline PPOP-2 & 920 & 4.29 & - & - & \\
\hline РPOP-3 & 880 & 3.24 & - & - & \\
\hline COF-JLU2 & 415 & 3.80 & 1.80 & 20.5 & S7 \\
\hline BDT-3 & 1010 & 2.9 & - & - & S32 \\
\hline BILP-6-NH ${ }_{2}$ & 1185 & 2.8 & - & - & S33 \\
\hline ТРМТР & 890 & 2.7 & - & - & 36 \\
\hline PCTF-4 & 1404 & 2.7 & - & - & 39 \\
\hline BILP-6 & 1261 & 2.7 & 1.90 & 13.2 & \multirow{2}{*}{$\mathrm{S} 34$} \\
\hline BILP-3 & 1306 & 2.4 & 1.70 & 16.6 & \\
\hline ALP-1 & 1235 & 2.6 & 1.50 & 20.8 & 91 \\
\hline Cz-POF-3 & 1927 & 2.54 & $1.71^{a}$ & 20.2 & S9 \\
\hline
\end{tabular}




\begin{tabular}{|c|c|c|c|c|c|}
\hline Cz-POF-1 & 2065 & 2.29 & $1.56^{a}$ & 19 & \\
\hline BILP-4 & 1135 & 2.53 & 1.80 & 13.0 & $\mathrm{~S} 4$ \\
\hline PPF-1 & 1740 & 2.43 & - & 15.1 & \multirow{2}{*}{89} \\
\hline PPF-2 & 1470 & 2.31 & - & 15.9 & \\
\hline BILP-12 & 1497 & 2.4 & 1.50 & 18.6 & S35 \\
\hline TNP-4 & 1348 & 2.38 & 1.42 & 20 & S36 \\
\hline MM2 & 1360 & 2.34 & $1.51^{b}$ & - & \multirow{2}{*}{68} \\
\hline MM1 & 1800 & 2.06 & $1.34^{b}$ & - & \\
\hline HEX-COF-1 & 1200 & 2.30 & 1.20 & 27 & $\mathrm{~S} 13$ \\
\hline APOP-3 & 1402 & 2.20 & 1.20 & 19.5 & \multirow{2}{*}{ S14 } \\
\hline APOP-1 & 1298 & 2.13 & 1.25 & 18 & \\
\hline CTF-TPC & 1668 & 2.14 & - & - & $\mathrm{S} 32$ \\
\hline PAF-60 & 1094 & 2.0 & 1.50 & 24.1 & S37 \\
\hline JUC-Z8 & 4743 & 2.0 & - & - & $\mathrm{S} 38$ \\
\hline
\end{tabular}

Table S9. Comparison of porosity and gas sorption properties of Tz- $d f$-CTFs with the current benchmark porous organic polymers.

\begin{tabular}{|c|c|c|c|c|c|c|}
\hline \multirow{2}{*}{$\begin{array}{l}\text { POP-based } \\
\text { Adsorbents }\end{array}$} & \multirow{2}{*}{$\frac{\begin{array}{c}\mathrm{S}_{\mathrm{BET}} \\
\left(\mathrm{m}^{2} \mathrm{~g}^{-1}\right)\end{array}}{77 \mathrm{~K}}$} & \multicolumn{2}{|c|}{$\begin{array}{c}\mathrm{CO}_{2} \text { Uptake } \\
{\left[\mathrm{mmol} \mathrm{g}^{-1}\right]}\end{array}$} & \multirow{2}{*}{$\begin{array}{c}\begin{array}{c}\mathrm{CH}_{4} \text { Uptake } \\
{[w \mathrm{t} \%]}\end{array} \\
273 \mathrm{~K}\end{array}$} & \multirow{2}{*}{\begin{tabular}{|c|}
$\begin{array}{c}\mathrm{H}_{2} \text { Uptake } \\
{[\mathbf{w t} \%]}\end{array}$ \\
$77 \mathrm{~K}$ \\
\end{tabular}} & \multirow[t]{2}{*}{ Ref. } \\
\hline & & $273 \mathrm{~K}$ & $298 \mathrm{~K}$ & & & \\
\hline Tz-df-CTF400 & 1550 & $6.42(2.32)$ & $4.32(1.33)$ & 3.63 & 2.51 & Our work \\
\hline Tz- $d f$-CTF500 & 1878 & $7.41(2.34)$ & $4.86(1.35)$ & 4.12 & 2.76 & Our work \\
\hline Tz-df-CTF600 & 2106 & $7.65(2.39)$ & $5.08(1.36)$ & 4.41 & 2.91 & Our work \\
\hline CQN-1g & 1870 & 7.16 & 4.57 & - & - & 95 \\
\hline NRPP-2 & 1028 & 7.06 & 3.71 & - & - & 94 \\
\hline HAT-CTFs & 1090 & $6.30(3.0)$ & $4.8(2.0)^{*}$ & - & - & 32 \\
\hline PPF-1 & 1740 & 6.12 & - & 2.43 & 2.75 & 89 \\
\hline CTF-py ${ }^{\mathrm{HT}}$ & 3040 & 5.97 & 4.22 & - & 2.63 & 96 \\
\hline CPOP-1 & 2220 & 4.82 & - & - & 2.80 & 98 \\
\hline TPC-1 & 1940 & 4.90 & - & 6.20 & 2.00 & 63 \\
\hline
\end{tabular}

*measured at $297 \mathrm{~K}$; values in parenthesis in mmol g-1 at 0.15 bar

Section Q. High Pressure $\mathrm{H}_{2}$ Adsorption Isotherms: 


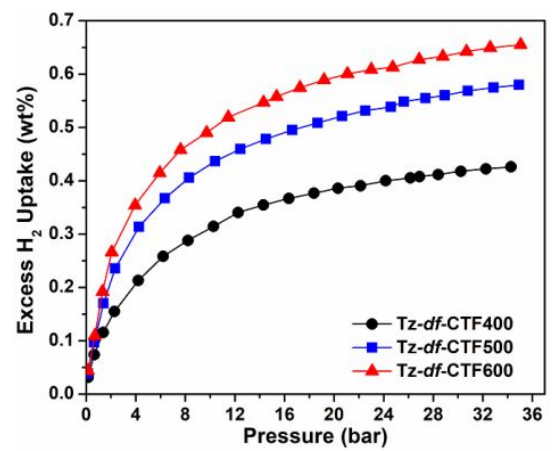

Figure S14. Excess $\mathrm{H}_{2}$ isotherms of Tz- $d f$-CTFs at $298 \mathrm{~K}$.

High-pressure (up to 35 bar) $\mathrm{H}_{2}$ adsorption measurements were also performed at $298 \mathrm{~K}$ and the resulting isotherms for Tz- $d f$-CTFs are presented in Figure S14 with uptake capacity up to $0.65 \mathrm{wt} \%$ (at $35 \mathrm{bar}, 298 \mathrm{~K}$ ) for Tz- $d f$-CTF600, which is higher than those of many bestknown porous materials under similar measurement conditions. ${ }^{58, \mathrm{~S} 39-\mathrm{S} 40}$

\section{Section R. Supporting References:}

S1: $\quad$ Tobe, Y.; Nakagawa, N.; Kishi, J.; Sonoda, M.; Naemura, K.; Wakabayashi, T.; Shida, T.; Achiba, Y. Polyyne Cyclization to form Carbon Cages: [16.16.16] $(1,3,5)$ Cyclophanetetracosayne Derivatives $\mathrm{C}_{60} \mathrm{H}_{6}$ and $\mathrm{C}_{60} \mathrm{Cl}_{6}$ as Precursors to $\mathrm{C}_{60}$ Fullerene. Tetrahedron 2001, 57, 3629-3636.

S2: $\quad$ Farha, O. K.; Yazaydın, A. Ö.; Eryazici, I.; Malliakas, C. D.; Hauser, B. G.; Kanatzidis, M. G.; Nguyen, S. T.; Snurr, R. Q.; Hupp, J. T. De Novo Synthesis of a Metal-Organic Framework Material Featuring Ultrahigh Surface Area and Gas Storage Capacities. Nature Chemistry 2010, 2, 944-948.

S3: $\quad$ Islamoglu, T.; Kim, T.; Kahveci. Z.; El-Kadri, O.M.; El-Kaderi, H. M. Systematic Postsynthetic Modification of Nanoporous Organic Frameworks for Enhanced $\mathrm{CO}_{2}$ Capture from Flue Gas and Landfill Gas. J. Phys. Chem. C 2016, 120, 25922599.

S4: Rabbani, M. G.; El-Kaderi, H. M. Synthesis and Characterization of Porous Benzimidazole-Linked Polymers and Their Performance in Small Gas Storage and Selective Uptake. Chem. Mater. 2012, 24, 1511-1517.

S5: $\quad$ Sekizkardes, A. K.; Altarawneh, S.; Kahveci, Z.; İslamoğlu, T.; El-Kaderi, H. M. Highly Selective $\mathrm{CO}_{2}$ Capture by Triazine-Based Benzimidazole-Linked Polymers. Macromolecules 2014, 47, 8328-8334.

S6: $\quad$ Wei, H.; Chai, S.; Hu, N.; Yang, Z.; Wei, L.; Wang, L. The Microwave-Assisted Solvothermal Synthesis of a Crystalline Two-Dimensional Covalent Organic Framework with High $\mathrm{CO}_{2}$ Capacity. Chem. Commun. 2015, 51, 12178-12181.

S7: Li, Z.; Zhi, Y.; Feng, X.; Ding, X.; Zou, Y.; Liu, X.; Mu, Y. An Azine-Linked Covalent Organic Framework: Synthesis, Characterization and Efficient Gas Storage. Chem. Eur. J. 2015, 21, 12079-12084.

S8: $\quad$ Chang, G.; Yang, L.; Yang, J.; Huang, Y.; Cao, K.; Ma, J.; Wang, D. A Nitrogen-Rich, Azaindole-Based Microporous Organic Network: Synergistic Effect of Local Dipole- $\pi$ and Dipole-Quadrupole Interactions on Carbon Dioxide Uptake. Polym. Chem. 2016, 7, 57685772. 

S9: Zhang, X.; Lu, J.; Zhang, J. Porosity Enhancement of Carbazolic Porous Organic Frameworks Using Dendritic Building Blocks for Gas Storage and Separation. Chem. Mater. 2014, 26, 4023-4029.

S10: Stegbauer, L.; Hahn, M. W.; Jentys, A.; Savasci, G.; Ochsenfeld, C.; Lercher, J. A.; Lotsch, B. V. Tunable Water and $\mathrm{CO}_{2}$ Sorption Properties in Isostructural AzineBased Covalent Organic Frameworks through Polarity Engineering. Chem. Mater. 2015, 27, 7874-7881.

S11: Puthiaraj, P.; Cho, S. -M.; Lee, Y. -R.; Ahn, W. -S. Microporous Covalent Triazine Polymers: Efficient Friedel-Crafts Synthesis and Adsorption/Storage of $\mathrm{CO}_{2}$ and $\mathrm{CH}_{4}$. J. Mater. Chem. A 2015, 3, 6792-6797.

S12: Yang, Y.; Zhang, Q.; Zhang, S.; Li, S. Synthesis and Characterization of Triphenylamine-Containing Microporous Organic Copolymers for Carbon Dioxide Uptake. Polymer, 2013, 54, 5698-5702.

S13: Alahakoon, S. B.; Thompson, C. M.; Nguyen, A. X.; Occhialini, G.; McCandless, G. T.; Smaldone, R. A. An Azine-Linked Hexaphenylbenzene Based Covalent Organic Framework. Chem. Commun. 2016, 52, 2843-2845.

S14: Song, W. -C.; Xu, X. -K.; Chen, Q.; Zhuang, Z. -Z.; Bu, X. -H. Nitrogen-Rich Diaminotriazine-Based Porous Organic Polymers for Small Gas Storage and Selective Uptake. Polym. Chem. 2013, 4, 4690-4696.

S15: Tian, Y.; Xu, S. -Q.; Qian, C.; Pang, Z. -F.; Jiang, G. -F.; Zhao, X. Two-Dimensional Dual-Pore Covalent Organic Frameworks Obtained From the Combination of Two $D_{2 \mathrm{~h}}$ Symmetrical Building Blocks. Chem. Commun. 2016, 52, 11704-11707.

S16: Xie, L. -H.; Suh. M. P. High $\mathrm{CO}_{2}$-Capture Ability of a Porous Organic Polymer Bifunctionalized with Carboxy and Triazole Groups. Chem. Eur. J. 2013, 19, 1159011597.

S17: Mondal, S.; Kundu, S. K.; Bhaumik, A. A Facile Approach for the Synthesis of Hydroxyl-Rich Microporous Organic Networks for Efficient $\mathrm{CO}_{2}$ Capture and $\mathrm{H}_{2}$ Storage. Chem. Commun. 2017, 53, 2752-2755.

S18: Modak, A.; Nandi, M.; Mondal, J.; Bhaumik, A. Porphyrin Based Porous Organic Polymers: Novel Synthetic Strategy and Exceptionally High $\mathrm{CO}_{2}$ Adsorption Capacity. Chem. Commun. 2012, 48, 248-250.

S19: Zhu, X.; Ding, S.; Abney, C. W.; Browning, K. L.; Sacci, R. L.; Veith, G. M.; Tian, C.; Dai, S. Superacid-Promoted Synthesis of Highly Porous Hypercrosslinked Polycarbazoles for Efficient $\mathrm{CO}_{2}$ Capture. Chem. Commun. 2017, 53, 7645-7648.

S20: Rabbani, M. G.; El-Kaderi, H. M. Template-Free Synthesis of a Highly Porous Benzimidazole-Linked Polymer for $\mathrm{CO}_{2}$ Capture and $\mathrm{H}_{2}$ Storage. Chem. Mater. 2011, 23, 1650-1653.

S21: Katsoulidis, A. P.; Kanatzidis, M. G. Phloroglucinol Based Microporous Polymeric Organic Frameworks with -OH Functional Groups and High $\mathrm{CO}_{2}$ Capture Capacity. Chem. Mater. 2011, 23, 1818-1824.

S22: Zhang, C.; Ying Liu, Y.; Li, B.; Tan, B.; Chen, C. -F.; Xu, H. -B.; Yang, X. -L. Triptycene-Based Microporous Polymers: Synthesis and Their Gas Storage Properties. ACS Macro Lett. 2012, 1, 190-193. 


\begin{tabular}{|c|c|}
\hline 23: & $\begin{array}{l}\text { Task-Specific Porous Organic Polymer Bifunctionalized with Carbazole and Triazine } \\
\text { oups. Chem. Commun. 2014, 50, 7933-7936. }\end{array}$ \\
\hline $24:$ & $\begin{array}{l}\text { Zhu, X.; Do-Thanh, C. -L.; Murdock, C. R.; Nelson, K. M.; Tian, C.; Brown, S.; } \\
\text { Mahurin, S. M.; Jenkins, D. M.; Hu, J.; Zhao, B.; Liu, H.; Dai, S. Efficient } \\
\mathrm{CO}_{2} \text { Capture by a 3D Porous Polymer Derived from Tröger's Base. ACS Macro Lett. } \\
\text { 2013, 2, 660-663. }\end{array}$ \\
\hline 25: & $\begin{array}{l}\text { Rabbani, M. G.; Sekizkardes, A. K.; El-Kadri, O. M.; Kaafarani, B. R.; El-Kaderi, H. M. } \\
\text { Pyrene-Directed Growth of Nanoporous Benzimidazole-Linked Nanofibers and their } \\
\text { Application to Selective } \mathrm{CO}_{2} \text { Capture and Separation. J. Mater. Chem. 2012, 22, 25409- } \\
25417 \text {. }\end{array}$ \\
\hline 26: & $\begin{array}{l}\text { u, S. -Y.; Mahmood, J.; Noh, H. -J.; Seo, J. -M.; Jung, S. -M.; Shin, S. -H.; Im, Y. - } \\
\text {; Jeon, I. -Y.; Baek, J. -B. Direct Synthesis of a Covalent Triazine-Based } \\
\text { camework from Aromatic Amides. Angew. Chem. Int. Ed. 2018, 57, 8438-8442. }\end{array}$ \\
\hline $\mathrm{S} 27$ & $\begin{array}{l}\text { Ren, S.; Bojdys, M. J.; Dawson, R.; Laybourn, A.; Khimyak, Y. Z.; Adams, D. J.; } \\
\text { Cooper, A. I. Porous, Fluorescent, Covalent Triazine-Based Frameworks Via } \\
\text { Room-Temperature and Microwave-Assisted Synthesis. Adv. Mater. 2012, 24, 2357- } \\
\text { 2361. }\end{array}$ \\
\hline S28 & $\begin{array}{l}\text { Puthiaraj, P.; Kim, S. -S.; Ahn, W. -S. Covalent Triazine Polymers Using a Cyanuric } \\
\text { Chloride Precursor Via Friedel-Crafts reaction for } \mathrm{CO}_{2} \text { adsorption/separation. Chem. } \\
\text { Eng. J. 2016, 283, 184-192. }\end{array}$ \\
\hline S29 & $\begin{array}{l}\text { Tao, L.; Niub, F.; Wanga, C.; Liuc, J.; Wanga, T.; Wang, Q. Benzimidazole } \\
\text { Functionalized Covalent Triazine Frameworks for } \mathrm{CO}_{2} \text { Capture. J. Mater. Chem. A } \\
\text { 2016, 4, 11812-11820. }\end{array}$ \\
\hline $\mathrm{S} 30$ & $\begin{array}{l}\text { Dey, S.; Bhunia, A.; Esquivel, D.; Janiak, C. Covalent Triazine-Based Frameworks } \\
\text { (CTFs) from Triptycene and Fluorene Motifs for } \mathrm{CO}_{2} \text { Adsorption. J. Mater. Chem. A } \\
\text { 2016, 4, 6259-6263. }\end{array}$ \\
\hline S31 & $\begin{array}{l}\text { Wang, T.; Zhao, Y. -C.; Zhang, L. -M.; Cui, Y.; Zhang, C. -S.; Han, B. -H. Novel } \\
\text { Approach to Hydroxy-Group-Containing Porous Organic Polymers from Bisphenol A. } \\
\text { Beilstein J. Org. Chem. 2017, 13, 2131-2137. }\end{array}$ \\
\hline S32 & $\begin{array}{l}\text { Bandyopadhyay, S.; Anil, A. G.; James, A.; Patra, A. Multifunctional Porous Organic } \\
\text { Polymers: Tuning of Porosity, } \mathrm{CO}_{2} \text {, and } \mathrm{H}_{2} \text { Storage and Visible-Light-Driven } \\
\text { Photocatalysis. ACS Appl. Mater. Interfaces 2016, 8, 27669-27678. }\end{array}$ \\
\hline S33 & $\begin{array}{l}\text { Islamoglu, T.; Behera, S.; Kahveci, Z.; Tessema, T. -D.; Jena, P.; El-Kaderi, H. M. } \\
\text { Enhanced Carbon Dioxide Capture from Landfill Gas Using Bifunctionalized } \\
\text { Benzimidazole-Linked Polymers. ACS Appl. Mater. Interfaces 2016, 8, 14648-14655. }\end{array}$ \\
\hline S34 & $\begin{array}{l}\text { Rabbani, M. G.; Reich, T. E.; Kassab, R. M.; Jackson, K. T.; El-Kaderi, H. M. High } \\
\mathrm{CO}_{2} \text { Uptake and Selectivity by Triptycene-Derived Benzimidazole-Linked Polymers. } \\
\text { Chem. Commun. 2012, 48, 1141-1143. }\end{array}$ \\
\hline S35 & $\begin{array}{l}\text { Sekizkardes, A. K.; İslamoğlu, A.; Kahveci, Z.; El-Kaderi, H. M. } \\
\text { Derived Benzimidazole-Linked Polymers to } \mathrm{CO}_{2} \text { Separation }\end{array}$ \\
\hline
\end{tabular}




\begin{tabular}{|c|l|}
\hline S36 & $\begin{array}{l}\text { Mondal, S.; Das, N. Triptycene Based 1,2,3-Triazole Linked Network Polymers } \\
\text { (TNPs): Small Gas Storage and Selective } \mathrm{CO}_{2} \text { Capture. J. Mater. Chem. A 2015, 3, } \\
\text { 23577-23586. }\end{array}$ \\
\hline S37 & $\begin{array}{l}\text { Yuan, Y.; Cui, P.; Tian, Y.; Zou, X.; Zhou, Y.; Sun, F.; Zhu, G. Coupling Fullerene into } \\
\text { Porous Aromatic Frameworks for Gas Selective Sorption. Chem. Sci. 2016, 7, 3751- } \\
\text { 3756. }\end{array}$ \\
\hline S38 & $\begin{array}{l}\text { Pei, C.; Ben, T.; Li, Y.; Qiu, S. Synthesis of Copolymerized Porous Organic } \\
\text { Frameworks with High Gas Storage Capabilities at Both High and Low Pressures. } \\
\text { Chem. Commun. 2014, 50, 6134-6136. }\end{array}$ \\
\hline S39 & $\begin{array}{l}\text { Panella, B.; Hirscher, M.; Pütter, H.; Müller, U. Hydrogen Adsorption in Metal- } \\
\text { Organic Frameworks: Cu-MOFs and Zn-MOFs Compared. Adv. Funct. Mater. 2006, } \\
\text { 16, 520-524. }\end{array}$ \\
\hline S40 & $\begin{array}{l}\text { Stadie, N. P.; Vajo, J. J.; Cumberland, R. W.; Wilson, A. A.; Ahn, C. C.; Fultz, B. } \\
\text { Zeolite-templated carbon materials for high-pressure hydrogen storage. Langmuir } \\
\text { 2012, 28, 10057-10063. }\end{array}$ \\
\hline
\end{tabular}

